\title{
Solar Dynamo Transitions as Drivers of Sudden Climate Changes
}

\author{
Silvia Duhau and Ernesto A. Martínez
}

Additional information is available at the end of the chapter

http://dx.doi.org/10.5772/51814

\section{Introduction}

There is a consensus about the origin of the increase of global surface temperature of the 20th century is the fast process of industrialization, that is producing an exponential increase in $\mathrm{CO} 2$ and other greenhouse gases in the boundary layer of the Earth atmosphere. However at 1924 a transition to a new configuration of the solar dynamo system occurred [1] that seated this system in the XX century Grand Maximum at which the highest values of solar activity of the last 400 years occurred. Therefore, the sharp increase of global temperature has been not only synchronic with the fast process of industrialization but also with a sudden increase of solar activity.

At the Schwabe polar cycle \#24 that started at year 2000 , maximized at year 2008 and would end at mid of 2013-14, a new solar dynamo transition is occurring that is leading to lower values of solar activity [2,3], and as a consequence the flux of solar energy on the Earth atmosphere is decreasing fast. Therefore by observing the future evolution of climate variables we will be able to evaluate the relevance of solar activity variability on climate changes.

A thorough determination of the contribution of solar activity to climate change is hindered by the fact that the only source of solar origin that is included in the climate models is total solar irradiance, TSI, for example see [4-8] This source of solar energy increased in the average in only about $0.13 \%$ along the last 400 years, which might explain at most a $30 \%$ [8] of the temperature increases along that period. However, besides TSI there are other sources of solar energy that might modify climate by mechanisms that have been proposed and studied by the last thirty years, for a review see $[9,10]$. The aim of the present work is to assess the impact of the 2008 dynamo transition on Earth's surface temperature. This will assist us to be well prepared in studying the unique experiment that nature is currently bringing to us, i.e. the solar dynamo transition and its consequences for climate that will be now documented worldwide and monitored with modern technical means. This may 
contribute to improve climate models by indicating the variables that are ignored in those models and that future observations finally prove to be relevant to climate changes.

The prediction of the impact of the 2008 solar dynamo transition on climate relies in the prediction of solar activity after the transition. Based in the regularities found in the time series of sunspot maxima along the last millennium Schove [11] predicted a value of sunspot maximum \#24 well below to those prevailing since 1924 and that fall near the low values that this cycle is having at only a year of its date of occurrence [12,13]

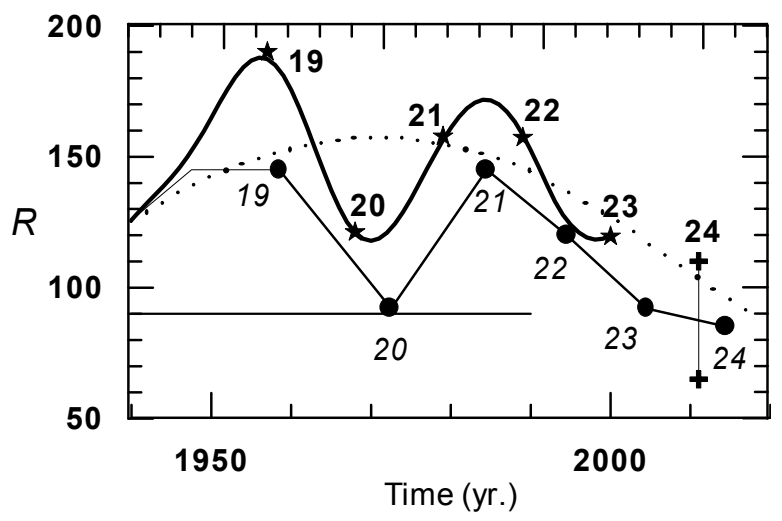

Figure 1. The predictions of Schove [11] for sunspot maxima \#19 to \#24 (filled circles) and the observed values (stars) together with an estimation of sunspot maxima 24 for the descending transition (crosses). The thick line is the envelope of sunspot cycle maxima. The constant level is the sunspot maxima average as determined prior 1923 and the dashed line is the secular variation in sunspot maxima time series for a descending transition (from [14]).

Unless in $[9,10,15]$ the only variable that is usually taken into account in the field of solarterrestrial physics for quantifying solar activity variability is sunspot number, of which direct observations does exist for the last 400 years. This variable gives a measure of the strength of the toroidal component of the solar magnetic field [16]. As solar activity ultimately depends on solar magnetic field strength [17-21] all the variables related to solar activity must bear some relationship with sunspot number. However, it was found [1, 14, 15, 22] that this relationship is non linear and so we need to resort to other proxies than sunspot number that give a better estimation of the true variables. These variables and its proxies are introduced in section 2 .

In our view, the success of Schove's [11] prediction of sunspot maximum \#24 by 50 years in advance, is based in the fact that being the solar dynamo a bounded system it undergoes natural modes of oscillation. However, the dynamics of this system is described by a set of non-linear differential equations, therefore its natural oscillations are non-stationary which impede us applying the Fourier base function to describe them. A mathematical methodology suitable for the description of natural oscillations in solar dynamo system has been developed by us $[2,3,15]$. This method and the way on which it is applied to predict sunspot maximum \#24 is briefly summarized in 3, and applied in section 4 to determining solar dynamo natural oscillations in the variables defined in 2 . The same method is applied in section 5 to look for 
the signatures of solar dynamo transition in surface temperature and from these results and the analysis of the latitudinal variation on temperature, that is presented in section 6, in section 7 the impact of the 2008 transition on the evolution of surface temperature along the XXI century is evaluated. Finally the conclusions are presented in section 8 .

\section{The solar dynamo transition in solar activity}

\subsection{The solar variables and its proxies.}

Solar activity has several manifestation, of these, those that are relevant to climate change are:

a. Total solar irradiation, TSI. This is the only source of solar origin that is considered in climate modeling, for example, see [5-9]. To study it here we resort to the reconstruction from Wang, Lean and Shelley [23-24].

b. Solar Flares produce the hard part of the electromagnetic spectrum (gamma and X rays and ultraviolet radiation) and also solar proton events. We resort to quantify it the Flare index . Data for this index [25] exist for the interval 1976.5-2008.5

c. Coronal Mass ejections, CME's, that are usually going together with flares and which main effect is the acceleration in the heliosphere-magnetosphere system of height energetic particles [26] leading, when interacting with the magnetosphere, to high energetic particles events. To quantify the geo-effectiveness of CME's we will resort to the Sudden Storm Commencement, SSC, index as defined by Duhau [27] that is based in the amplitude and rise time of geomagnetic storm sudden commencement, defined by Mayaud [28], and computed in the interval 1868-1998 from the ISGI data [29] .

d. Variability of the solar coronal magnetic field, the solar 'open flux' that modulates cosmic ray particles flux. The solar open flux is well described by the geomagnetic index aa [30] defined by Mayaud [31]. The data in the interval 1844-1985 is from [32] and from 1986 onward there are two version of this index, one is the standard from ISGI [29] and the other is the data from [33].

Solar activity ultimately depends on solar magnetic field variations, that has two components: the toroidal and the poloidal one [17-21]. Since now on we will call 'strength' of the polaidal and toroidal field to the amplitude of the Schwabe cycle on the respective solar magnetic field component.

The sunspot number gives a measure of the total toroidal field at the spots [16]. Therefore sunspot number at solar maximum, Rmax, is a measure of the strength of this component. While at solar minimum the polar field is mainly dipolar and the open flux is coming mostly from this field, as a result geomagnetic index aa at solar minimum, aamin, gives a measure of the dipolar field of the Sun [2, 15, 34-37]

Rmax is determined here from the yearly values of Group Sunspot Number [38] and of sunspot number International time series value as provided by NOAA [39] for the intervals 1610-1704 and 1705-2011 respectively. Aamin is determined from the two data sets of geomagnetic index aa as detailed in paragraph (d). Note that while aa index is always a proxy for the solar open flux, only at solar minimum is also a proxy for the strength of the dipolar component of the global magnetic field. 


\subsection{The solar dynamo transition as seen in the solar variables.}

Rmax, and aamin fluctuated around constant levels of 93, 4 spots and $10.3 \mathrm{nT}$ respectively [1]. We have called these couple of constants 'transition point', that is a point in the 'phase diagram' of Rmax vs. aamin (as proxies for the toroidal and polar magnetic field strengths) (Figure 2). The transition point has the property that when the path of successive points in this diagram is close enough to it, a transition occurs in the solar dynamo system that leads to a sudden change in the strength and length of its natural oscillations.

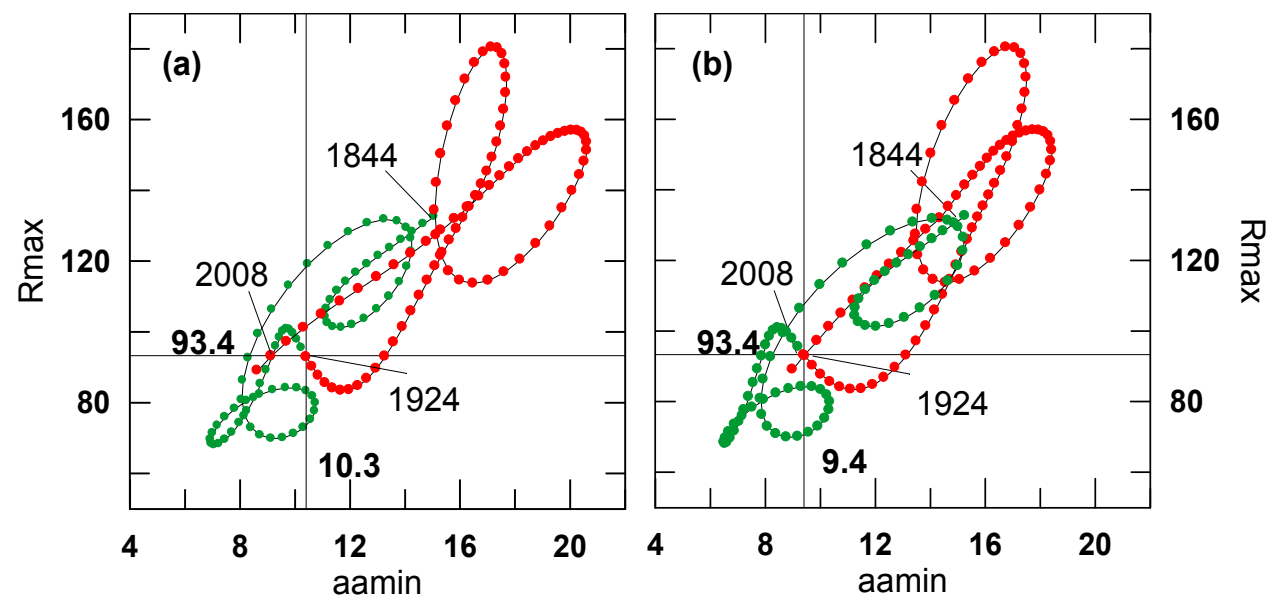

Figure 2. The diagram for the period 1844-2011 per year for (a): the Standard data from ISGI and (b) the Lockwood homogenized time series data (from [37]).

There are three types of solar dynamo episodes: Grand Maximum, Grand Minimum and Regular oscillations. Which of then occur after a transition depends on how close to the transition point is passing the path when coming back to that point. The paths determined by the two different data sets of Figure 2, passed exactly (within the experimental error) by the transition point at 1924 and the Grand Maximum Episode of the XX century (red points) started. The same happened at the 2008 transition for the data at the right, which indicates that a Gran Minimum is coming after this transition. But for the data at the left the path at 2008 is far enough from the transition point to lead to predict that a Regular Oscillations type episode, alike to that occurring prior 1924 (green points), would instead follows after the transition. From the properties of the oscillations in proxy time series of Rmax from Schove [11] and of Usoskin [40] for the last 1700 years, we [37] have estimated that, among the two possible type of episode that may occurs after a Grand Maximum, the forthcoming episode would be of a Regular type, alike to the one previous to 1924 (green points in Figure 5 ) and that this episode would endure for the rest of the present millennium.

A full sequence of the three types of episodes occurred since 1610 [1]. The solar dynamo transitions produces a sudden change in the average value of the successive maxima of the Schwabe, 11 year, cycle (Figure 3), that occurs in synchronicity in all the related variables. 
This indicates that a substantial change in solar dynamo configuration occurs after each transition that is affecting all the layers that compose the solar dynamo system.

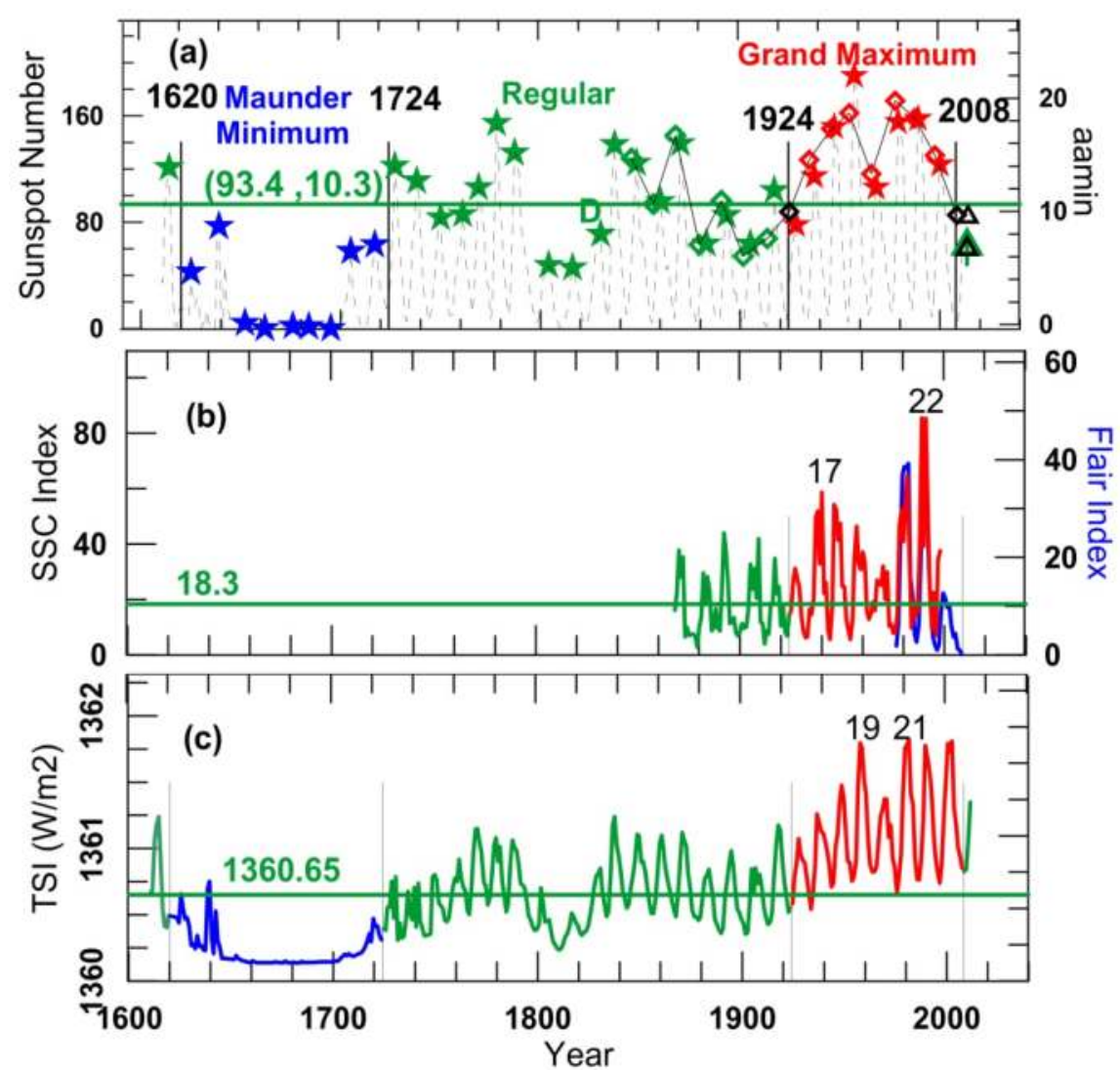

Figure 3. Solar dynamo transitions in (a) sunspot number maxima (stars) and geomagnetic index at minima (diamonds). The black diamonds indicates the polar cycles at which the transitions occur and the horizontal green line the transition point (93.4 spot, $10.4 \mathrm{nT}$ ) level [1]. The letter D indicates the short type Dalton Minimum. The green and black triangles are the predictions from [11] (upper black),[2] (green) and [3] (lower black), respectively. (b): SSC Index and Flare Index (blue line). (c): total solar irradiance, TSI. In (b) and (c) the horizontal green lines are at the average value along the Regular Oscillations episode and the black numbers are the conventional numbering of the strongest maxima occurring after 1924.

It may be observed that at and above the secular time scale all the variables have a very similar behavior, and so the corresponding oscillations appear having the same length and 
nearly the same phase. The same do not happen with oscillations with shorter length, as much as for example, the two relative maxima that occurs in all the variables after 1924 transition are not synchronic, but are at solar cycle maxima \#17 and \#22, in SSC and Flare Indexes (Figure 3b), and at solar cycle maxima \#19 and \#21 in TSI (Figure 3c). This is studied further in the section 4 and 5 by means of the mathematical methodology that is discussed in the next section.

The effect of CME's and flares on the heliosphere-magnetosphere system is generically called 'solar storm'. It is know that both phenomena are often going together and in fact Flare index, along the short interval on which is known, follows closely to SSC index that is a proxy for the geo effectiveness of CME's. Therefore since now we will consider SSC as an acceptable proxy for the geo-effectiveness of solar storms.

\section{A base function of compact support for representing solar dynamo natural oscillations}

The Fourier spectrum of sunspot number time series, see [41] and references therein, have prominent peaks. The most conspicuous among them, like the Gleissberg, the Süess and the Hallstatt one, are usually called 'cycles'. However, we do not know a priori the real nature of the phenomena that leads to these peaks and its variability. As much as to call cycle to any new peak that appear in the spectra of solar variables has been called by Hoyt and Schatten [42] 'cycle mania'.

We have introduced a methodology that allows splitting solar activity related time series in natural oscillations, based in a study of the variability on time of the peaks in the spectra and a suitable base function to represent the time series in real time, as is summarized below.

\subsection{Natural modes of oscillation in the wavelet spectrum.}

It is apparent in the time series of Figure 2, natural modes of oscillations in a non-liner non-stationary system, like the solar dynamo system, have a transitory behavior which make it impossible representing them by the discrete Fourier base function, that presupposes that the waves are linear and stationary. As the solar dynamo system is bounded we still may apply a discrete transform method, but due to the transient nature of its natural oscillations the applied base function must be of compact support [43], as are the wavelet base function. The shape of the selected wavelet must be alike to that of the signal that is being represented [44]. The wavelet spectrum of the Rmax time series (see Figure 4) has peaks with a Gaussian envelope that indicates the presence of oscillations with fairly well defined periodicities. On the other hand, the variables as a function of time (Figure 3) exhibit oscillation with rapid time changes in their amplitude. Therefore we have selected the Morlet wavelet base function that is a harmonic function with a Gaussian envelope. 
The wavelet spectrum of sunspot number time series and its proxies have peaks at periods that goes from seconds, to millenniums. As the time series analyzed here is Rmax, oscillations with lengths below the Hale cycle length ( 22 years) are not contained in the times series. Besides the time changes in amplitude of all the peaks on the spectrum, at and below the lower Gleissberg band, the Fourier period of the dominant peaks changes strongly on time too, as an example of this we have computed the spectra (Figure 4) for two different time interval.

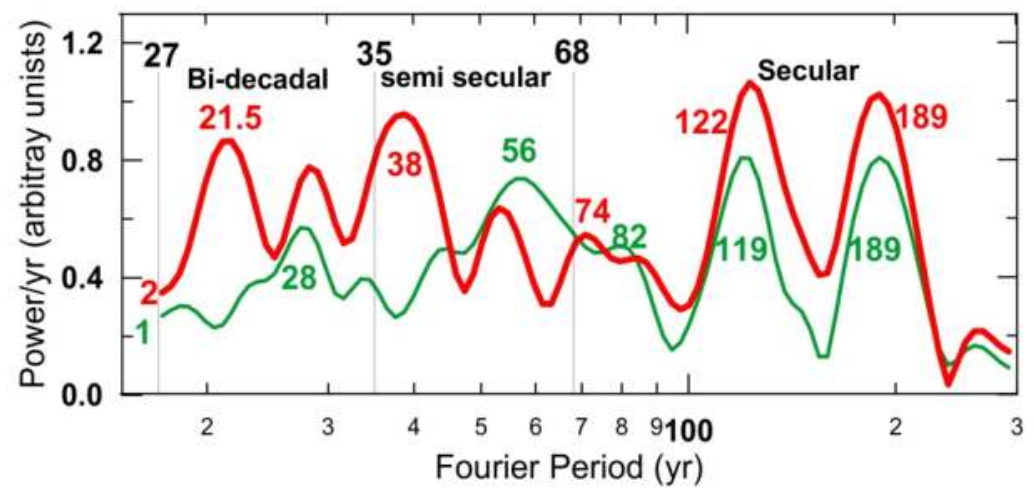

Figure 4. The Morlet wavelet spectrum for two time spans: 1665- 1865 (1) , 1865-2000 (2). The vertical bars delimitates the Fourier periods of the wavelet components that are included in each of the three period bands. The colored numbers are the values of the Fourier periods of the nearest peak. Below the 90 year period, only the most prominent peak within a given period band is indicated, for each time span .

For each of the spectra on Figure 4, the Fourier period of the dominant peak in the bidecadal band is the first quasi-harmonic of the Fourier period of the dominant peck in semisecular band. As the Fourier period of the dominant peaks change from a time interval to the other, the length of the corresponding natural oscillation changes too. This is further analyzed next.

\section{Time changes of the natural modes of oscillations.}

We will study here only the solar variables that are relevant to climate change and that has a proxy time series larger than a century, these are TSI, aamin, that is proxy for the open flux strength and SSC, that is a proxy for the frequency and intensity of solar storms. As a result of the analysis summarized in 3 we have split the time series in three oscillations (Figure 5). These oscillations are found by adding all the wavelets components which Fourier periods are in the respective bands as defined in Figure 4. In the case of the secular oscillation the linear trend is added and the transition level is subtracted. A preliminary interpretation of the phenomena underlying the three oscillations is given in [3]. 


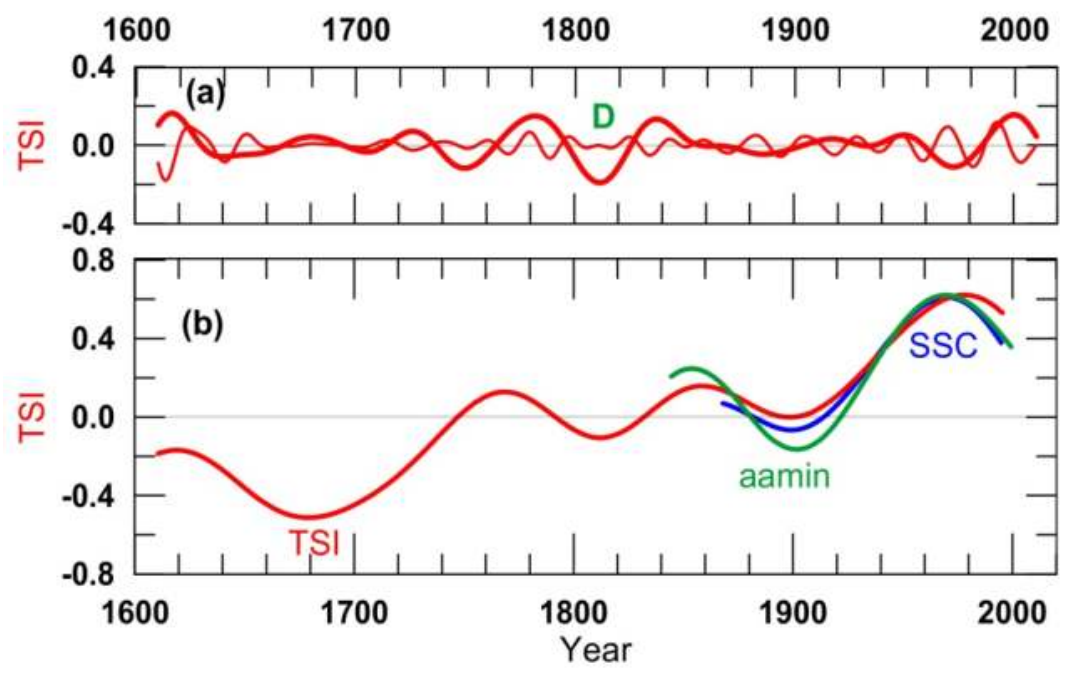

Figure 5. The three solar dynamo natural oscillations (a): The bi decadal (thin line) and the semi secular (thick line) in TSI. (b) the secular oscillations in TSI, aamin and SSC, were SSC and aamin are scaled to fit TSI at the secular oscillation that produced the XX century Grand Maximum.

The short Dalton Minimum that occurred around 1820 is the result of the synchronization of a relative minimum in a strong semi-secular (Figure 5a) occurring in synchromicty with a relative minimum in a weak secular (Figure $5 b$ ) oscillations. Note that while the semi secular oscillation around de Dalton minimum has a length of about 60 years, it has a length of only 40 years along the Grand Maximum (Figure5a). Therefore a increases of the amplitude of the semi-secular oscillation is synchronic with a increases of its length. This behavior dramatically occurs in the secular oscillation (Figure $5 b$ ).

On base of the relationship between the length of the oscillations and its intensity we have predicted the date of occurrence of solar maxima \#24, to be at 2013.5 [2]. And in base of the time changes of the three oscillations we have predicted the value of sunspot maximum \#24. After a descending transition, like the 2008, either a Grand Minimum or a Regular Oscillations episode (blue and green starts in Figure 6) may occur. These two possible cases are barely distinguishable between them This is due to the fact that sunspot maximum \#24 would occur at 2013.5 [2,12] and the secular oscillation passed by cero only at 2008 and so this oscillation will have only six years and a haft to develop. Only by sunspot maximum \#25 the two cases will differentiate unambiguously [37]

The sequence \#23-\#24 is found to be alike to the -\#13-\#12 and also to the \#11-\#12 ones (see numbered maxima in Figure 6). These three sequences are similar but no equal, since a 
given sequence never repeat identically due to the variability in relative phase and amplitude of the three natural oscillations on solar dynamo system.

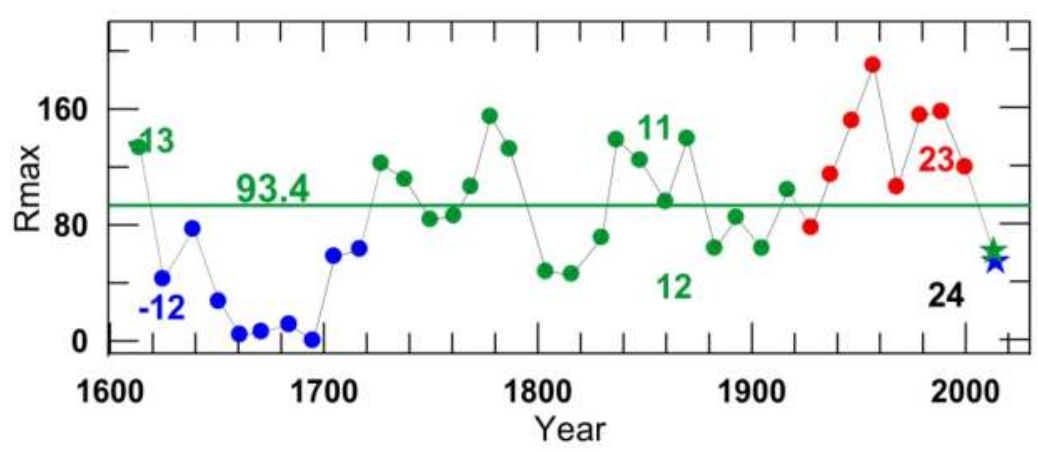

Figure 6. Rmax time series (the data are the same that in Figure 3a) and the predicted values for sunspot cycle \#24, for the Regular episode [2] and for the Grand Minimum [3] cases (blue and green stars, respectively). The two sequences of sunspot maxima that are alike to the \#23-\#24 sequence are indicated in the figure by its conventional numbering. To assign them prior the Dalton Minimum we have assumed that the sunspot cycle that occurred prior the \#5 was lost in the sunspot number time series, as suggested in [45]

\section{Solar dynamo transition as seen in the Earth surface temperature}

To find the signature in Earth surface temperature of the sudden changes on solar dynamo natural oscillations we represent the time series of north hemisphere temperature for the last 400 years by the same base function that we have used when representing the natural oscillations in solar variables in section 4 . By adding wavelet components with periodicities above the 17 years we include (Figure 7) the three natural modes of modes of oscillations in solar activity and at the same time we filter the natural oscillations in the climate system.

The temperature increases since 1610 has not been steady but occurred in four steps (blue, green, red and violet horizontal lines in figure 7), three of them occurred in synchronicity with the solar dynamo transition, and the fourth, that is seen in the surface data (black line) but not in the satellite data (blue line), started at 1970. The fact that each of the first three steps occurred in synchronicity with the date of occurrence of the solar dynamo transition suggest that the increase of temperature is linked to some solar variables that have had sudden increases after each transition. This appears to be the case of solar storms (see its proxy, SSC, in Figure 3b). Moreover, the last step follows the sudden increases of solar storms that started at 1950 and reached a value that quadruplicates those prior 1924 at sunspot maximum \#22, peaking at 1989. This is studied further in the following by analyzing the relationship between the secular and the semi secular oscillation in temperature with that in the solar variables. 
The secular variation is alike in all the solar variables (see fig $5 \mathrm{~b}$ ). As a result, if solar activity were the main source of climate changes the secular oscillation in temperature must be alike to the secular oscillation in any of the variables related to solar activity. Only one of the four relevant variables, TSI, have a time series long enough to test this similitude. The result is in Figure 8. There is a good agreement between the secular oscillation in TSI and temperature, more if we take into account that the secular oscillation in the other involved variables (Figure $5 b$ ) has a similar but not an equal time variation.

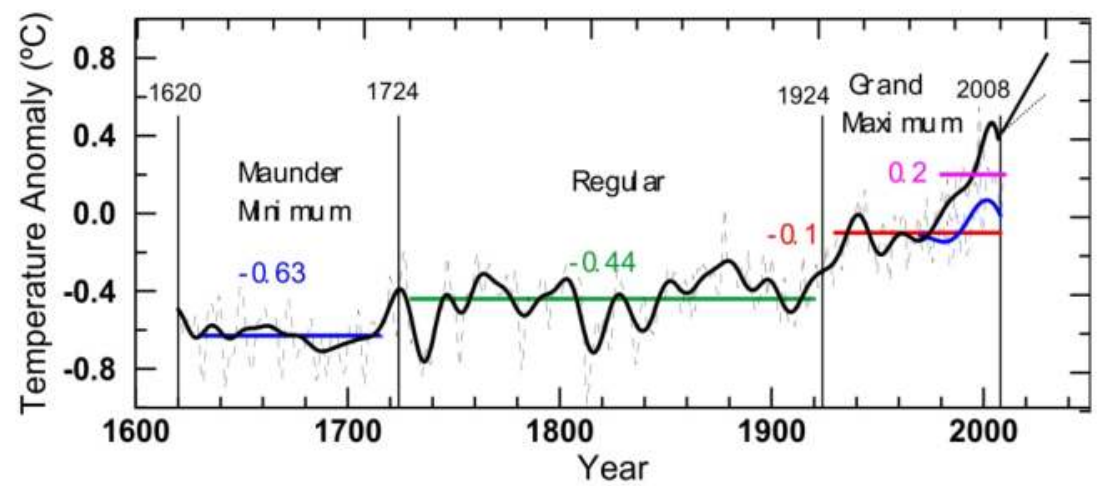

Figure 7. Solar dynamo transitions in global surface temperature. The dashed black line is the yearly averages of paleodata [46] in the interval 1610-1849 to which ground based data [47,48] from 1850 onward are pasted (dashed black line) The dashed blue line is satellite UAH MSU lower troposphere temperature data [49] and the thick line are the smoothed values (see text). The vertical lines indicate the dates of occurrence of the four historically documented solar dynamo transition (cf. black diamonds in Figure 3a). The projections of IPCC [50] for the forthcoming twenty years are shown for two cases: one on which the emissions of greenhouse gases would continue at the same rate as today (full) and the other on which it would remain in the actual level (dashed black line) .

The secular oscillation in surface temperature (Figure 8) started increasing above the secular oscillation in TSI at 1860 and reached a value that is $0.3^{\circ} \mathrm{C}$ above the one expected from the secular oscillation in TSI. We cannot jump immediately to the conclusion that this departure is entirely due to the industrial revolution, because it was not gradual, but occurred mostly in the interval 1860-1900 and after 1980, that is after the last two relative maxima in the secular oscillation in solar activity that occurred at 1957 and 1977 (see Figure 5b).

A recovery from the little ice age (LIA) is occurring since 1800-1850 [51, 52]. And, after the mid-1970s, ice mass loss has accelerated [52-54]. This is consistent with the suggestion [9-10] that the rapid increases of surface temperature along the XX century Grand Maximum was due to the increases in the frequency and intensity of solar storms which geo-effectiveness (see figure 3b), increased suddenly after the 1924 transition to quadruplicate its value prior 1924 at sunspot cycle 22 maxima, peaking at 1990.

The semi -secular oscillation in temperature and TSI (Figure 9) are alike. It is the strongest in SSC. After 1924 the semi secular oscillation in all the variables has two relative maxima, one prior and the other after 1970, like it happened with temperature (see also Figure 7). 


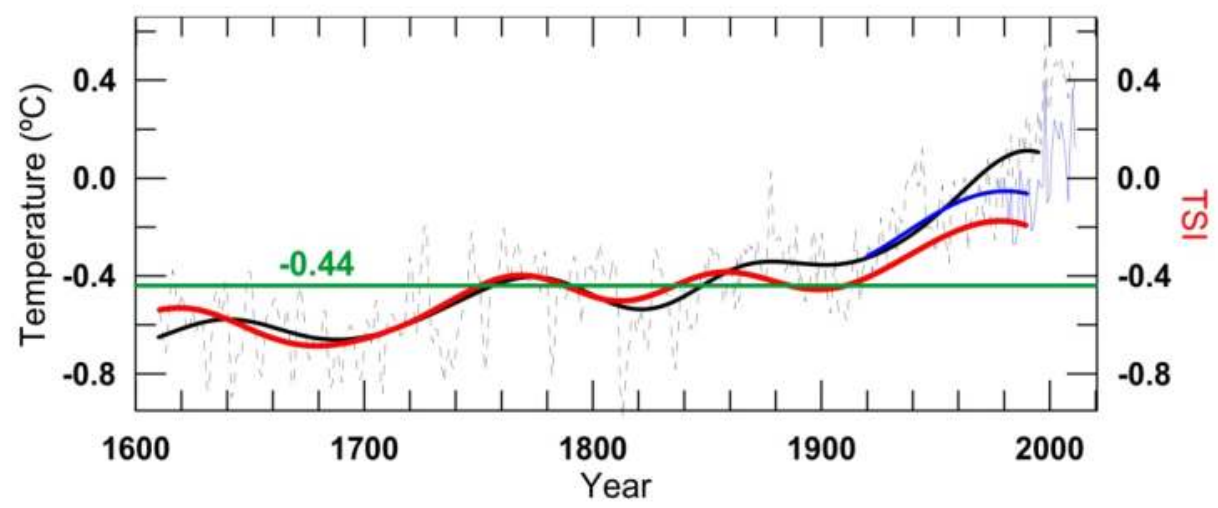

Figure 8. The secular oscillation in the temperature data of Figure 7. and in TSI of Figure 3c, this last scaled to fit temperature prior 1800.

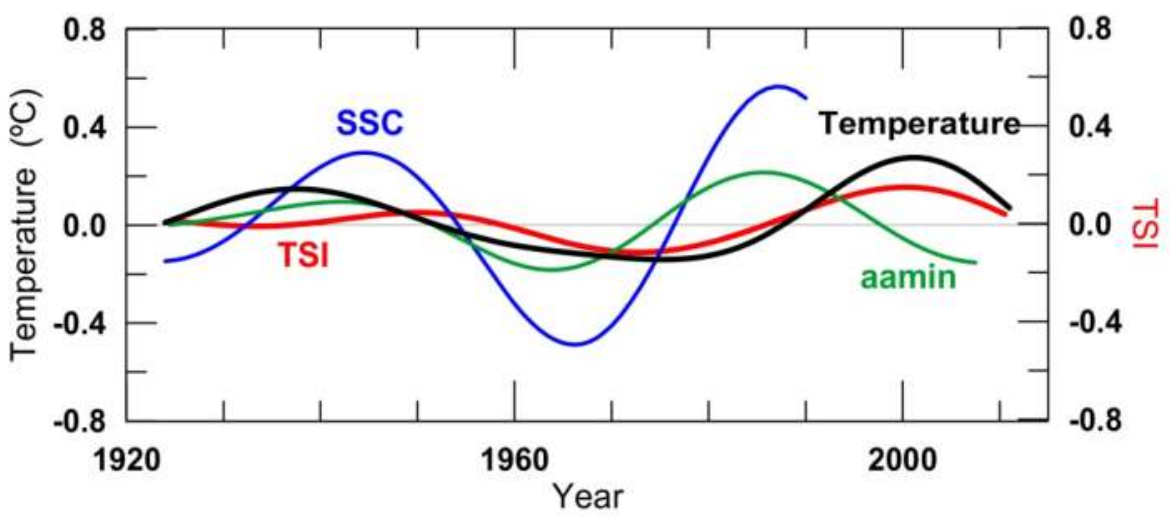

Figure 9. The semi secular oscillation in surface temperature and in the three relevant solar variables as indicated in the figure. TSI, SSC and aamin are scaled by the same factor that in Figure 8.

\section{An analyses of the latitudinal variation on temperature}

There is a hiatus in average global temperature increase is recent years. This may be the first indication of the impact on climate of current decreases of solar activity. However, at latitudes below $24^{\circ}$ (see Figure10d) the fast increases that started at all latitudes at 1970 , is still going on. 


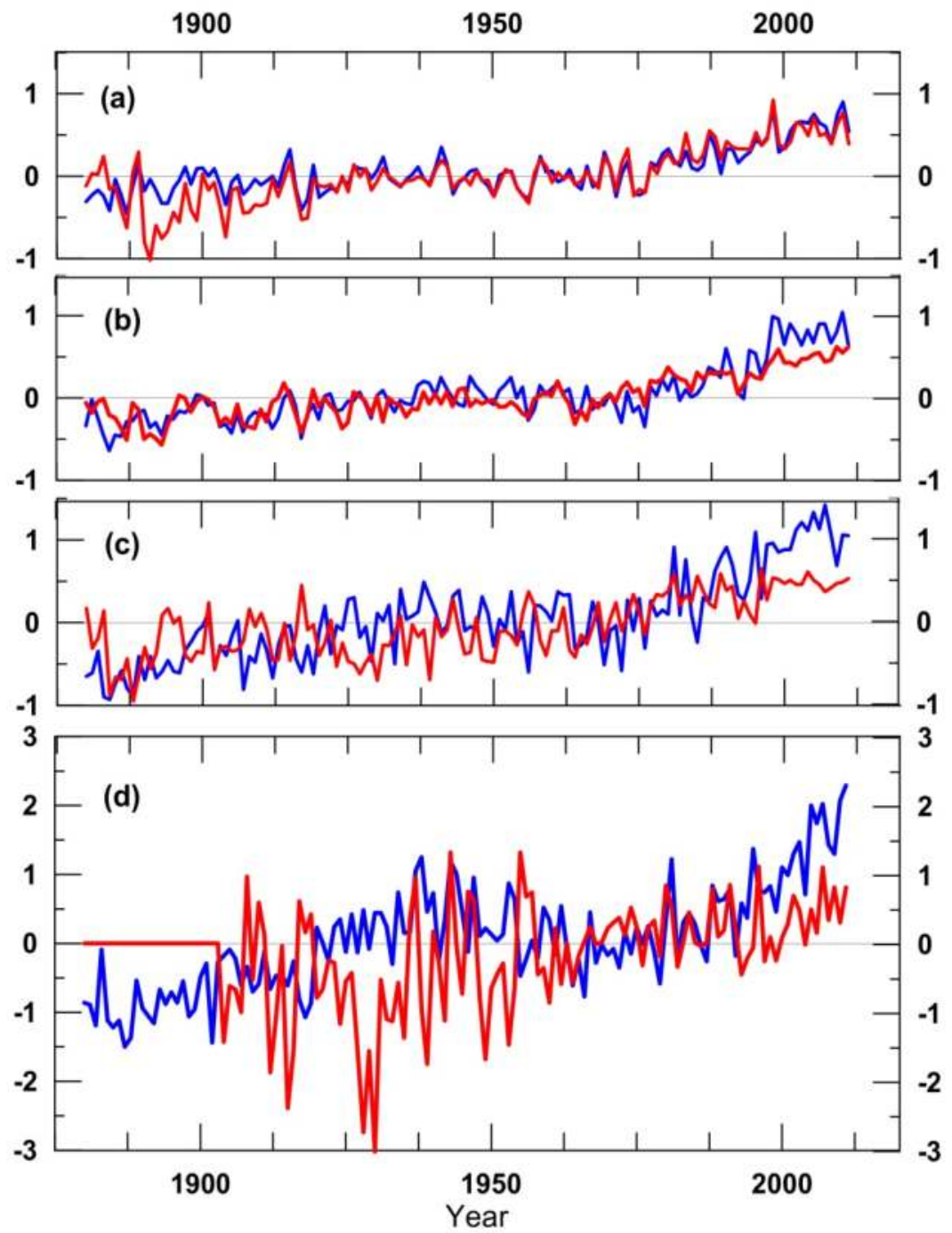

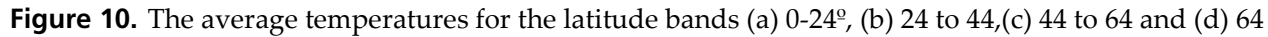
to 90 , for the south (red) and the north (blue) , hemisphere, respectively . The data is from [55]

Ice melting has accelerated since 1980 [52-54] and some models indicates that the ice feedback albedo mechanism due to this acceleration is appreciable at the poles [56] and also at height latitudes [57]. This feedback mechanism is still not well represented in IPCC 
climate models that underestimate Arctic see ice thinning by a factor of 4 and fail to capture the recent see ice kinematic acceleration [53]. From this we may conclude that, either icealbedo feedback is underestimated in the IPCC models [54], or some source of heating mainly operating in the polar cap and at high latitudes is missing in those models. The synchronicity between ice kinetic acceleration with the occurrence of the strongest solar storms (Figure 3b and Figure 9 blue line), that after 1970, in only 20 years duplicates its strength, is consistent with this hypothesis, because these solar events, when interacting with the Earth magnetosphere operates mainly in the polar cap and at the subauroral region [58-62]. The mechanism involved are reviewed and some evidences that a $70 \%$ of global temperature increases after the 1924 transition was due to this strong increases on solar storms are presented in $[9,10]$.

If the above source of heating were significant, they may explain the step like appearance of the temperature time series (Figure 7) if we assume that not only after the 1924 transition, that is the only one along which a proxy, SSC, for the geoeffectiveness of solar storms is known, but also after any solar dynamo ascending transition, solar storms substantially increases in less that 25 years, while it takes to TSI (compare blue and red lines in Figure 9) by more than 40 years to reach its first relative maximum. At that moment, following its semi-secular oscillation the strength of solar storms has already decreased and therefore the principal source of heating at high latitudes is fading out. However, the ice is still melted and the secular oscillation in TSI is still at its maximum, so the temperature is maintained at the same levels than before due to enhancement of the TSI heating by positive ice-albedo feedback.

Solar dynamo is well over its cycle \#24 and the average value of TSI (Figure 3c) is still well above the average prior 1924. The decrease of TSI (red line in figure 9) is being delayed with respect to the decreases of the other solar variables. As was delayed its increases when emerging the Sun form the Maunder Minimum at 1724 (ccompare Figure 3c with Figure 3b). By the contrary, solar storms (see figure $2 b$ ), has already started decreasing by 20 years ago and will fall to a half of its 1990 values (sunspot maxima 21, see Figure 3b) by 2013.5, if the above mechanism sustain surface temperature would start decreasing in the near future. In the next section we present an estimation of this decrease.

\section{An estimation of the future evolution of surface temperature}

There are sources of climate change other than anthropogenic gases and solar activity that are:

1. Natural oscillations of the climate system and volcanism. These sources vary in the bidecadal time scale and below [5]. To filter then we have taken into account oscillations in the semi-secular time scale and beyond.

2. Time changes in the strength of the dipolar component of the geomagnetic field and its tilt angle, that is the position of the geomagnetic poles, that modulates the geoeffectiveness of solar storms in the atmosphere [58-62]. Appreciable changes in geomagnetic dipolar field strength and its tilt angle occurs in time scales at and above 
the millennial one [63-65]. As a result the contribution to climate change of geomagnetic field along the current century may be disregarded.

3. It has been suggested $[66,67]$ that periods of acceleration of the Earth's rotation rate correspond to years of increasing intensity of the zonal circulation and to global-surface warming, and periods of deceleration correspond to years of decreasing zonal-circulation intensity and to a global decrease in surface temperatures. A measure of the acceleration of the Earth's rotation rate is provided by the time derivative of the excess of the length of the day, LOD. This variable follows solar activity with a delay of 94 years [68] and so along the time interval analyzed in $[66,67]$ that is 1850 to 1960 , the semi secular oscillation in LOD was the strongest of the last 400 years, because it was the one around the Dalton Minimum episode. Only along this exceptionally strong semi secular oscillation LOD has appreciably contributed to temperature [10] , and so we may disregard its contribution to the secular and the semi secular oscillation along the XXI century.

We conclude that the main sources of global warming at the XXI century will be the industrial increase of greenhouse gases and solar activity. In view of the inability of climate models in reproducing present ice melting acceleration and the mechanism that we have presented here by which solar activity increases might explain this phenomena, there are some possibility that the strong increases in solar activity after the 1924 transition was the principal driver of the climate warming of the XX century. However, as a careful evaluation of this mechanism is still lacking we can not rule out the possibility that, by that contrary most of the atmospheric heating of the XX century was of anthropogenic origin.

In the case that solar activity was the source of the decreases of global temperature along the XX century, global temperature will start decreasing not later than about 2020 when solar dynamo will be fully settled in its new Grand Episode. It has taken by 150 years for ice melting reaching current levels. After the 1924 transition the increases on solar storms from the values sustained along the Regular oscillation episodes occurred in two steps, one culminating at 1947, and the other at 1989, but solar storms has already decreased to values comparables to those along the Regular episode, that is at $25 \%$ of its value around 1989 in only one step, and the decreases in TSI to its values prior 1924 will culminates by 2024 , as a result we expect that it will take unless a century to ice cover to be at the same level than prior 1850 and so, as solar activity will continue with the moderate levels corresponding to a Regular Oscillation episode the temperature would be oscillating around the same level than prior 1923 , that is $-0.44{ }^{\circ} \mathrm{C}$, by the end of the present century.

In the other hand, in the case that most of the warming since 1970 were of anthropogenic origin the contribution of solar activity to temperature change would be of $-0.34{ }^{\circ} \mathrm{C}$. As for the next two decades [50] a increases of about $0.2^{\circ} \mathrm{C}$ per decade is projected for a range of SRES emission scenarios. Even if the concentrations of all greenhouse gases and aerosols were kept constant at year 2000 levels, a further warming of about $0.1^{\circ} \mathrm{C}$ per decade would be expected. Therefore the heating due to greenhouse gases along the forthcoming 20 years will be between $0.2^{\circ} \mathrm{C}$ to $0.4^{\circ} \mathrm{C}$ and so the effect of solar activity would lead to the temperature stay constant or decreasing at the most in $0.14{ }^{\circ} \mathrm{C}$ till about 2030 to start increasing again after that, unless greenhouse emission were severely limited in the future. 


\section{Conclusions}

The sudden increases of solar activity that occurred after the 1724 and 1924 solar dynamo transitions, has been accompanied by a sudden increases of average surface temperature of $0,2^{\circ} \mathrm{C}$, and $0.34^{\circ}$ after 1974 and 1924 , respectively. Therefore, of the total increases of the average temperature level, that was of $\sim 0.8^{\circ} \mathrm{C}$ along the last 400 years, less than $0.3^{\circ}$ may be of non solar origin, in agreement with previous results [15]

A solar dynamo transition to a new Grand Episode of lower solar activity is occurring, that would be settled at sunspot cycle \#24 [1, 14, 15]. It would be alike [37] to the 1724-1924 Regular Oscillations episode. In fact sunspot cycle maximum \#24, that would occur at 2013.5, is being the weakest of the last 100 hundred years [13], being alike to sunspot cycle maximum \#12 occurring at 1883.

Wile greenhouse gases emission continued increasing at present, there is a hiatus in temperature increases since 10 years ago. This may be the first indication of the impact of the current solar dynamo transition on climate cooling. However, at latitudes above the $64^{\circ}$ (North and South) temperature it is still increasing fast as much as there is a acceleration of the ice-melting since 1980 [52-54] and some models indicates that the ice feedback albedo mechanism due to this acceleration is appreciable at the poles [56] and also at height latitudes [57]. This may indicates that this feedback mechanism is still not well represented in IPCC climate models, since they underestimate Arctic sea ice thinning by a factor of 4 and fail to capture the recent sea ice kinematic acceleration [54]. Or it may indicate that a heating source that mainly operates at the polar cap and high latitudes is still missing in those models, and, as suggested in $[9,10]$ this source may be solar storms, which after the mid1970s have duplicated their average intensity and frequency as compared with those occurring at the XIX century.

Natural sources of climate changes has been reviewed here, and from present knowledge of them it was concluded that in the long term (time scales above the semi-secular) the main sources of climate change along the past century were greenhouse gases and solar activity, and that the same would happen along the current, XXI century. The episode of Regular Oscillations in solar activity that is starting by now would endure for the rest of the present millennium [37]. If this prediction and the principal source of polar and high latitudes atmosphere heating were solar storms, the sudden decreases of the geoeffectiveness of solar storms to a $50 \%$ of its values prevailing along the XX century Gran Maximum will lead to global temperature to decreases from the present average level of $\sim 0.2^{\circ} \mathrm{C}$ to the same level that along the 1724-1924 Regular Oscillation episode, that is $-0.44^{\circ} \mathrm{C}$. The time that it will take to the climate system to react to current decreases of solar activity sensitively depends on the ice-albedo feedback mechanism that is still not well known. An estimation of this time is possible by observing that is has taken $\sim 150$ for the recovering from the Little Ice Age $[51,52]$. The fact that the principal solar source of atmosphere heating at the poles and high latitudes has already decreased to its values prevailing prior 1924, allows us estimating that the expected decreases in $0.64^{\circ} \mathrm{C}$ would occurs in about 100 years. Solar storms has decreased yet to values alike to that prevailing at the XIX century, but TSI will decreases 
substantially only by sunspot cycle maximum \#25 (that will occur at $\sim 2024$ ), a appreciable decreases of temperature would be observed only by 2030 .

In the case that the main source of heating of the last 100 years were greenhouse gases, the solar activity decreases would contribute with a cooling of only $\sim 0.3^{\circ} \mathrm{C}$, and taking into account the projections of temperature from climate models [50], the sudden decreases of solar activity that is going on would mitigate the impact of greenhouse gases on global warming only by the forthcoming 20 years.

\section{Author details}

Silvia Duhau

Departamento de Física, Facultad de Ingeniería, Universidad de Buenos Aires and Consejo Nacional de Investigaciones Científicas y Técnicas, Argentina

Ernesto A. Martínez

Dirección General de Cultura y Escuelas, Buenos Aires, Argentina

\section{References}

[1] Duhau, S., De Jager, C. The solar dynamo and its phase transitions during the last millennium. Solar Phys. 2008; 250, 1.

[2] De Jager, C., Duhau, S. Forecasting the parameters of sunspot cycle 24 and beyond. J. Atm. Solar Terr. Phys. 2007; 71, 239.

[3] Duhau, S., De Jager C. The forthcoming Grand Minimum of solar activity. J. of Cosmology 2010; 8, 1983.

[4] Kiehl, J. T., Hack, J. J., Bonan, G. B. , Boville, B. A. Williamson, D. L., Rasch, P. J. The National Center for Atmospheric Research Community Climate Model: CCM3*. J. Climate 1998; 11, 1131.

[5] Solomon, S., Manning, D.J., Qin, M , Chen, Z., Marquis, M., Averyt, K.B., Tignor M. and Miller, H.L. (eds.). IPCC. Contribution of Working Group I to the Fourth Assessment Report of the Intergovernmental Panel on Climate Change, Chapter 8 Climate Models and Their Evaluation. Cambridge University Press, Cambridge, United Kingdom and New York, NY, USA 2007; http://www.ipcc.ch/pdf/assessment-report/ar4/wg1/ar4-wg1chapter8.pdf (accessed 07 May 2012).

[6] Dwyer, J., Norris J. R. and Ruckstuhl, C. Do climate models reproduce observed solar dimming and brightening over China and Japan? . J. of Geophys; Res. 2010; 115, D00K08, 8 PP., 2010 , doi:10.1029/2009JD012945.

[7] Kopp, G. and Lean, J. L. A new, lower value of total solar irradiance: Evidence and climate significance. Geophys. Res. Lett. 2011; 38, L01706, 7 PP., doi: 10.1029/ 2010GL045777.

[8] Lean, J., and Rind, D. Evaluating Sun-climate relationships since the Little Ice Age . J. of Atmosph. and Solar-Terr. , Phys. 1999; 61, 25. 
[9] Duhau, S. Long Term Variations in Solar Magnetic Field, Geomagnetic Field and Climate, Procceding of $9^{\text {th }}$ Asian-Pacific Regional IAU (APRIM 2005) edited by Sutantyo, W., Premadi, P. W., Mahasena, P., Hidayat, T. and Mineshige, S. 2005; 18.

[10] Duhau, S. Solar activity, Earth's rotation rate and climate variations in the secular and semi-secular time scales. Phys. and Chemistry of the Earth. 2006; 31, 99.

[11] Schove, D. J. The sunspot cycle, 649 B.C. to A.D. 2000. J. of Geophys. Res. 1955; 60, 127.

[12] Hathaway, D. A Standard Law for the Equatorward Drift of the Sunspot Zones. Solar Phys. 2011; 273, 221, DOI 10.1007/s11207-011-9837.

[13] Marshal Space Flight center. http://solarscience.msfc.nasa.gov/predict.shtml (accessed 02 July 2012)

[14] Duhau S. An early prediction of sunspot maximum 24, Solar Phys. 2012; 213, 203, DOI: 10.1023/A:1023260916825.

[15] De Jager, C. and Duhau, S. The variable solar dynamo and the forecast of solar activity; effects on terrestrial surface temperature; in J. M. Cossia (ed), Proceedings of the global warming in the 21th century. NOVA science publishers, Hauppauge, NY, 2010; 77.

[16] Nagovitsyn Y. To the description of long-term variations in the solar magnetic flux: The sunspot area index. Astron. Lett. 2005; 31, 557. Translated from Pis'ma v Astronomicheski ${ }^{1}$ Zhurnal, 31, No. 8, 622.

[17] Fisher, C.H., Fan, Y., Longcope, B.W., Linton, H.G., Pevtsov, A.A. The Solar Dynamo and Emerging Flux - (Invited Review), Solar Phys. 2000; 192, 119

[18] Tobias, S.M. The solar dynamo . Phil. Trans. R. Soc. Lond, A 2002; 360, 2741; DOI1.1078/rsta.2000.1090.

[19] Ossendrijver, MUnderstanding the solar dynamo, Astron. Astrophys. Rev. ., 2003; 11, 287.

[20] Dikpati M., de Toma, G. and Gilman, P. A. Predicting the strength of solar cycle 24 using a flux-transport dynamo-based tool, Geophis. Res. Lett. 2006; 33, L05102, doi:10.1029/2005GL025221.

[21] Charbonneau, P. Dynamo models of the solar cycle, Living Rev. Solar Phys. 2012; 7. tirl:http://solar physics.livingreviews.org/Articles/lrsp-2010-3/. (accessed 20 January 2012).

[22] Duhau S. and Chen, C. The sudden increase of solar and geomagnetic activity after 1923 as a manifestation of a non-linear solar dynamo, Geophys. Res. Lett. 2002; 29, 10.1029/2001GL013953.

[23] Wang, Y. M, Lean, J. L. and Sheeley, N.R. . Jr. Modeling the Sun's Magnetic Field and Irradiances since1713. The Astrophysical Journal 2005; 625, 522.

[24] The laboratory for Atmospheric and Space Physics. http://lasp.colorado.edu/sorce/tsi_data/TSI_TIM_Reconstruction.txt (accessed 01 , May 2012).

[25] The Geophysical Data Center (ftp://ftp.ngdc.noaa.gov/STP/SOLAR_DATA/SOLAR_FLARES/FLARES_INDEX/Yearly/ (accessed 01 May 2012). 
[26] Lario D. and Simnett. G. M. Solar Energetic particle Variations, in Solar Variavility and its Efects on Climate, J. M. Papaand P. F. Fox Eds. AGU, Geophysical Monograh 2003; 141, 195.

[27] Duhau, S. Global Earth surface temperature changes induced by mean Sun dynamo magnetic field variations In: Solar variability as an input to the Earth's environment. International Solar Cycle Studies (ISCS) Symposium, 23 - 28 June 2003, Tatranská Lomnica, Slovak Republic. Ed.: A. Wilson. ESA SP-535, Noordwijk: ESA Publications Division, ISBN 92-9092-845-X, 2003; 317.

[28] Mayaud, P.N. Analysis of storm sudden commencements for the years 1868-1967. J. Geophys. Res. 1975; 80 (A1): doi:10.1029/0JGREA0000800000.

[29] Service International des Indices Geomagnetiques, ISGI publication Office http://isgi.latmos.ipsl.fr/lesdonne.htm (accessed 02 January 2012).

[30] Lockwood, M., Stamper, R. and Wild, M. N. A Doubling of the Sun's Coronal Magnetic Field during the Last 100 Years. Nature. 1999; 399, 437.

[31] Mayaud, P. N. The aa indices: A 100-year series characterizing the magnetic activity, J. Geophys. Res. 1972; 72, 6870..

[32] Nevanlinna, H., and Kataja, E.: An extension of the geomagnetic index series aa for two solar cycles (1844-1868). Geophys. Res. Lett. 1993; 20; 2703.

[33] Lockwood, M., D.. Hancock, W., B , Henwood, R. Ulich, R. , Linthe, H. J., Clarke, E. and Clilver, A. M. The long-term drift in geomagnetic activity: calibration of the aa index using data from a variety of magnetometer station , 2006;

(http://www.eiscat.rl.ac.uk/Members/mike/publications/pdfs/sub/241_Lockwood_aa_co rrect_S1a.pdf (accessed 02 January 2012)

[34] Legrand, J. P. and Simon, P. A. A two component solar cycle, Solar Phys. 1991; 121, 187.

[35] Russell, C. T., On the possibility of delivering interplanetary and solar parameters from geomagnetic records, 1975. Solar Phys., 42, 259.

[36] Layden, A. C., Fox, P. A., Howard, J. M., Dsarajedini, K. H. and Sofia, S. Dynamo based scheme for forecasting the magnitude of solar activity cycle. Solar Phys. 1991; 132, 140.

[37] De Jager C. and Duhau, S. Sudden transitions and grand variations in the solar dynamo, past and future, J. Space Weather Space Clim. 2012; 2 , A07, DOI: $10.1051 /$ swsc/2012008.

[38] Hoyt, D. V., and Schatten, K. Group sunspot numbers: A new solar activity reconstruction. Solar Phys. 1998; 179, 189.

[39] Naval Research laboratory. ftp://ftp.ngdc.noaa.gov/STP/ SOLAR_SUNSPOT_NUMBERS/AMERICAN/X (accessed 01 May 2012).

[40] Usoskin I. G. , Solanki, S., Schüssler, M., Mursula. K., Alanko, K. Millennium-scale sunspot number reconstruction; evidence for an unusually active sun since the 1940s. Phys. Rev. Lett. 2003; 91, NO 21, 211101-1.

[41] De Jager, C. Solar forcing of climate. 1. Solar variability. Space Sci. Rev. 2005; 120, 197.

[42] Hoyt, D. V. and Schatten, K. The Role of the Sun in Climate Change, Oxford University Press.1997. 
[43] Farge, M. Annu. Rev. Fluid Mech. 1992. Wavelet transforms and their applications to turbulence 1992, 24, 395.

[44] Torrence, C., Compo, G. P. A Practical Guide to Wavelet Analysis. Bull. Amer. Meteor. Soc. $1998 ; 79,61$.

[45] Usoskin I., Mursula G. K. and Kovaltsov, G. A. Lost sunspot cycle in the beginning of Dalton minimum: New evidence and consequences Geophys. Res. Lett. 2002; 29, 2183, doi:10.1029/2002GL015640, 2002

[46] Moberg, M., Sonechkin, D. M., Holmgren, K.. Datsenko N. M. and Karlén, W. Highly variable Northern Hemisphere temperatures reconstructed from low- and highresolution proxy data Nature 2005; 433, 613.

[47] Jones, P. D., Lister, D. H., Osborn, T. J., Harpham, C. Salmon, M., Morice, C. P. Hemispheric and large-scale land-surface air temperature variations: An extensive revision and an update to 2010. J. of Geophys. Res. 2012; 117, D05127, 29 PPdoi:10.1029/2011JD017139.

[48] University of West Anglia, Climate Research http://www.cru.uea.ac.uk/cru/data/temperature/hadcrut3nh.txt (accesed 02 January 2012).

[49] NOAA http://vortex.nsstc.uah.edu/data/msu/t2lt/uahncdc.lt (accessed 02 January 2012).

[50] Solomon, S., Manning, D.J., Qin, M , Chen, Z., Marquis, M., Averyt, K.B., Tignor M. and Miller, H.L. (eds.). IPCC. Contribution of Working Group I to the Fourth Assessment Report of the Intergovernmental Panel on Climate Change, Projections of futre change in climate, Cambridge, United Kingdom and New York, NY, USA. 2007;

http://www.ipcc.ch/publications_and_data/ar4/wg1/en/spmsspm-projections-of.html (accessed 15 July 2012).

[51] Akasofu, S. On the recovery from the Little Ice Age, Natural Sciences 2011; 2, 1211.doi:10.4236/ns.2010.211149.

[52] Masiokas , M. H., Luckman , B. H., Villalba , R., Ripalta , A., Rabassa, J. , Little Ice Age fluctuations of Glaciar Río Manso in the north Patagonian Andes of Argentina, Quaternary Res. 2010; 73, 96.

[53] Stroeve, J., Holland, M. M., Meier, W. Scambos, T. and Serreze, M. Arctic sea ice decline: Faster than forecast, Geophs. Res. Lett. 20016; 34, L09501, 5 PP., doi:10.1029/2007GL029703.

[54] Rampal P., Weiss, J., Dubois, C and , Campin, J. M. IPCC Climate models do no capture ice arctic sea ice drift acceleration : consequences in term of projected sea ice thinning and declining, J. Geophys. Res. 116, C00D07, 17PP.

[55] NASA GSFC http://data.giss.nasa.gov/gistemp (assecced 02 May 2012).

[56] Perovich, D., Light, B., Eicken, H., Jones, K. F., Runciman K. and Nghiem, S. V. Increasing solar heating of the Arctic Ocean and adjacent seas, 1979-2005: Attribution and role in the ice-albedo feedback. Geophsy. Res. Lett. 2007; 34, L19505, 5 PP.doi:10.1029/2007GL031480.

[57] Austin, J. and Coleman, S. M: Lake Superior summer water temperatures are increasing more rapidly than regional air temperatures: A positive ice-albedo feedback. Geophys. Res. Lett. 2007; 34, L06604, doi:10.1029/2006GL029021. 
[58] Bucha, V. Conclusions. In: Magnetic Field and the Processes in the Earth's Interior, Ed.in- Chief V. Bucha, Co-edited by G. Petrova, S. Burlatskaya, I Cupal, V. P. Golovkov, H. Kautzleben and W. Webers., Prague Academia, 1983.

[59] Bucha, V. and Bucha, V. Jr. Geomagnetic forcing of changes in climate and in the atmospheric circulation, J. of Atmosph. and Solar Terr. Phys. 1998; 60, 146.

[60] Jackman, Ch. H. and MacPeters, R. D. The effect of solar proton events on ozone and other constutents. In: Solar Variability and its Effects on Climate , J. Pap and P. Fox, eds. Geophysical Monograph, 141, American Geophys. Union, 2005; 305.

[61] Pudovkin M. I. and Morozova, A. L. Time variation of atmospheric pressure and circulation associated with temperature changes during Solar Proton Events, J. of Atmos. and Solar Terr. Phys. 1998; 60, 1729.

[62] Morozova A. L. , Pudovkin M. I. and Thejll, P. Variations of atmospheric pressure during solar proton vents and Forbush decreases for different latitudinal and synoptic zones. Int. J. of Geomagnetism and Aeronomy 2002; 3, 181.

[63] Wilson R. L. Dipole Offset-The Time-Average Palaeomagnetic Field Over the Past 25 Million Years, Geophys. J. of the Royal Astron. Soc. 1971; 22, 491.

[64] Yang, S., Odah H., and Shaw, J. Variations in the geomagnetic dipole moment over the last 12,000 years Geophys. J. Int. 2000; 140, 158.

[65] Korte, M and Constable, C. G. Continuous geomagnetic field models for the past 7 millennia: 2. CALS7K Geochemistry Geophysics. Geosystems 2005, 6, Q02H16, 18 PP., 2005doi:10.1029/2004GC000801.

[66] Lambeck, K. and Cazenave, A. Long Term Variations in the Length of Day and Climatic Change Geophys. J. R. Astr. Soc. 1976; 46, 555.

[67] Hunt, N. B. G. The effects of past variations of the Earth's rotation rate on climate Nature 1979, 281, 188; doi:10.1038/281188.

[68] Duhau S. and Martinez, E. On the origin of the fluctuations in the length of day and in the geomagnetic field on a decadal time scale Geophys. Res. Lett. 1995; 22, 3283. doi:10.1029/95GL03285. 